\title{
ON THE MODIFIED MOD $p$ LOCAL LANGLANDS CORRESPONDENCE FOR $\mathrm{GL}_{2}\left(\mathbb{Q}_{\ell}\right)$
}

\author{
DAVID HELM
}

\begin{abstract}
We give an explicit description of the modified $\bmod p$ local Langlands correspondence for $\mathrm{GL}_{2}\left(\mathbb{Q}_{\ell}\right)$ of [3], Theorem 5.1.5, where $p$ is an odd prime different from $\ell$.
\end{abstract}

\section{Introduction}

In [3], Matthew Emerton and the author introduce a "modified mod $p$ local Langlands correspondence," a "mod $p$ " version of the local Langlands correspondence that is well-behaved under specialization and has useful applications to the cohomology of modular curves and the "local Langlands correpondence in families" of [3]. Section 5 of [3] gives a general characterization of this mod $p$ correspondence in terms of its basic properties. If one restricts to the group $\mathrm{GL}_{2}$, it is easy in most cases to go from this list of characterizing properties to an explicit description of this correspondence. These easy cases are discussed in detail in section 5.2 of [3]. When $p$ is odd the cases discussed come close to a complete description of the correspondence, but omit certain more difficult special cases. The purpose of this note is to explicitly describe the correspondence in these more difficult cases and thus complete the description of the modified $\bmod p$ local Langlands correspondence for $\mathrm{GL}_{2}$ and odd $p$.

Let $\ell$ be a prime, and let $F$ be a finite extension of $\mathbb{Q}_{\ell}$ whose residue field has order $q$. (It seems likely that one can relax this assumption, and let $F$ be an arbitrary local field with residue field $\mathbb{F}_{q}$, but [3] does not consider these cases and so we will not either.) Let $p$ be a prime distinct from $\ell$, and let $k$ be a finite field of characteristic $p$. The modified mod $p$ local Langlands correspondence is an association $\bar{\rho} \mapsto \bar{\pi}(\bar{\rho})$, where $\bar{\rho}: G_{F} \rightarrow \mathrm{GL}_{n}(k)$ is a continuous $n$-dimensional representation of the absolute Galois group of $F$, and $\bar{\pi}(\bar{\rho})$ is a finite length indecomposable smooth representation of $\mathrm{GL}_{n}(F)$. Its interest arises from its nice behaviour under specialization, which we discuss below, and also from the fact that it arises "in nature" in the cohomology of the tower of modular curves. Indeed, in [2], Emerton considers the following situation:

Denote by $X(N)$ the modular curve with full-level $N$ structure, and let $X(N)_{\overline{\mathbb{Q}}}$ be its base change to a curve over $\overline{\mathbb{Q}}$. Let $\Sigma$ be a finite set of primes containing $p$, and let $H_{\Sigma}^{1}$ be the direct limit:

$$
\lim _{\operatorname{supp} N \subseteq \Sigma} H_{e ́ t}^{1}\left(X(N)_{\overline{\mathbb{Q}}}, k\right) .
$$

(Here $\operatorname{supp} N$ denotes the set of primes dividing an integer $N$, so the limit is over $N$ divisible only by primes in $\Sigma$, ordered by divisibility.) The space $H_{\Sigma}^{1}$ acquires actions

Received by the editors October 5, 2012.

2000 Mathematics Subject Classification. 11F70 (Primary), 22E50 (Secondary). 
of $G_{\mathbb{Q}}$, of $\mathrm{GL}_{2}\left(\mathbb{Q}_{p}\right)$, and of $\mathrm{GL}_{2}\left(\mathbb{Q}_{\ell}\right)$ for $\ell \neq p$, as well as of the Hecke operators $T_{r}$ for $r \notin \Sigma$ and the diamond operators $\langle d\rangle$ for $d$ not divisible by any prime of $\Sigma$. Let $\mathbb{T}_{\Sigma}$ be the subalgebra of $\operatorname{End}_{k}\left(H_{\Sigma}^{1}\right)$ generated by these Hecke operators and diamond operators.

Let $\bar{\rho}: G_{\mathbb{Q}} \rightarrow \mathrm{GL}_{2}(k)$ be a modular Galois representation unramified outside $\Sigma$. Then there is a maximal ideal $\mathfrak{m}$ of $\mathbb{T}_{\Sigma}$ attached to $\bar{\rho}$, and (under certain hypotheses on the local behaviour of $\bar{\rho}$ at $p$ ), Emerton has shown ([2], Theorem 6.2.13 and Proposition 6.1.20) that the $\mathfrak{m}$-torsion $H_{\Sigma}^{1}[\mathfrak{m}]$ of $H_{\Sigma}^{1}$ is a product of "local factors":

$$
H_{\Sigma}^{1}[\mathfrak{m}] \cong \bar{\rho} \otimes \pi_{p} \otimes \bigotimes_{\ell \neq p, \ell \in \Sigma} \pi_{\ell}
$$

where $\pi_{p}$ is attached to $\left.\bar{\rho}\right|_{G_{\mathbb{Q}_{p}}}$ by considerations from the $p$-adic Langlands program (see [2], section 3 , for details) and each $\pi_{\ell}$ is the representation $\bar{\pi}\left(\left.\bar{\rho}\right|_{G_{\mathbb{Q}_{\ell}}}\right)$ attached to the restriction of $\bar{\rho}$ to a decomposition group at $\ell$ via the modified $\bmod p$ local Langlands correspondence for $\mathrm{GL}_{2}\left(\mathbb{Q}_{\ell}\right)$. Thus an explicit description of the modified mod $p$ local Langlands correspondence for $\mathrm{GL}_{2}\left(\mathbb{Q}_{\ell}\right)$ gives an explicit description of the action of $\mathrm{GL}_{2}\left(\mathbb{Q}_{\ell}\right)$ on the cohomology of the modular tower.

We now recall more precisely the definition of the modified $\bmod p$ local Langlands correspondence. The starting point is the characteristic zero "generic local Langlands correspondence" of Breuil-Schneider [1]. We refer the reader to sections 4.2 and 4.3 of [3] for the basic properties of this correspondence. In particular, this correspondence associates to any $n$-dimensional Frobenius-semisimple Weil-Deligne representation $(\rho, N)$ over a field $K$ containing $\mathbb{Q}_{p}$ an indecomposable (but often reducible) admissible representation $\pi(\rho, N)$ of $\mathrm{GL}_{n}(F)$. If instead we have a Galois representation $\rho: G_{F} \rightarrow \mathrm{GL}_{n}(K)$, we define $\pi(\rho)$ to be the representation $\pi\left(\rho^{\mathrm{ss}}, N_{\rho}\right)$, where $\left(\rho^{\mathrm{ss}}, N_{\rho}\right)$ is the Weil-Deligne representation attached to $\rho$.

The representations $\pi(\rho, N)$ have several nice properties. In particular, they are essentially $A I G$ representations, a concept introduced in Section 3.2 of [3]. A smooth representation $\pi$ of $\mathrm{GL}_{n}(F)$ over a field $K$ is called essentially AIG if

- the socle of $\pi$ is absolutely irreducible and generic,

- $\pi$ contains no generic irreducible subquotients other than its socle, and

- $\pi$ is the sum of its finite length submodules.

Such representations have several useful properties. In particular, their only endomorphisms are scalars ([3], Lemma 3.2.3), any submodule of an essentially AIG representation is essentially AIG, any non-zero map of essentially AIG representations is an embedding, and such an embedding, if it exists at all, is unique up to a scalar factor ([3], Lemma 3.2.2). Moreover, if $\pi$ is an absolutely irreducible generic representation of $\mathrm{GL}_{n}(F)$, then there is an essentially AIG representation $\operatorname{env}(\pi)$, known as the essentially AIG envelope of $\pi$, such that the socle of $\operatorname{env}(\pi)$ is isomorphic to $\pi$ and any essentially AIG representation $\pi^{\prime}$ with socle isomorphic to $\pi$ embeds in $\operatorname{env}(\pi)$ ([3], Proposition 3.2.7). Moreover, all the subquotients of env $(\pi)$ (or, more generally, of any essentially AIG representation) have the same supercuspidal support ([3], Corollary 3.2.14).

A final useful property of essentially AIG representations is that they contain distinguished lattices (up to homothety). In particular, let $\mathcal{O}$ be a discrete valuation ring 
with residue field $k$ and field of fractions $K$, and let $\pi$ be an essentially AIG representation over $K$. Suppose further that $\pi$ is $\mathcal{O}$-integral; that is, contains a $\operatorname{GL}_{n}(F)$ invariant $\mathcal{O}$-lattice. Then there is an $\mathcal{O}$-lattice $\pi^{\circ}$ in $\pi$, unique up to homothety, such that $\pi^{\circ} \otimes_{\mathcal{O}} k$ is essentially AIG ([3], Proposition 3.3.2).

This last property is crucial because it allows for a definition of the modified mod $p$ local Langlands correspondence via "compatibility with reduction mod $p$ " from the characteristic zero correspondence of Breuil-Schneider. More precisely, one has:

Theorem 1.1 ([3], Theorem 5.1.5). Let $k$ be a finite field of characteristic $p$. There is a map $\bar{\rho} \mapsto \bar{\pi}(\bar{\rho})$ from the set of isomorphism classes of continuous representations $G_{F} \rightarrow \mathrm{GL}_{n}(k)$ to the set of isomorphism classes of finite length admissible smooth $\mathrm{GL}_{n}(F)$-representations over $k$, uniquely determined by the following three conditions:

(1) For any $\bar{\rho}$, the associated $\mathrm{GL}_{n}(F)$-representation $\bar{\pi}(\bar{\rho})$ is essentially $A I G$.

(2) If $K$ is a finite extension of $\mathbb{Q}_{p}$, with ring of integers $\mathcal{O}$ and residue field $k^{\prime}$ containing $k, \rho: G_{F} \rightarrow \mathrm{GL}_{n}(\mathcal{O})$ is a continuous representation lifting $\bar{\rho} \otimes_{k} k^{\prime}$, and $\pi^{\circ}$ is the unique $\mathcal{O}$-lattice in $\pi\left(\rho \otimes_{\mathcal{O}} K\right)$ such that $\pi^{\circ} \otimes_{\mathcal{O}} k^{\prime}$ is essentially $A I G$, then there is an embedding

$$
\pi^{\circ} \otimes_{\mathcal{O}} k^{\prime} \hookrightarrow \bar{\pi}(\bar{\rho}) \otimes_{k} k^{\prime} .
$$

(3) The representation $\bar{\pi}(\bar{\rho})$ is minimal with respect to satisfying conditions (1) and (2); that is, for any $\bar{\rho}$, and any representation $\pi$ satisfying these conditions with respect to $\bar{\rho}$, there is an embedding of $\bar{\pi}(\bar{\rho})$ in $\pi$.

The construction of $\bar{\pi}(\bar{\rho})$ is straightforward. One shows that for a given $\bar{\rho}$, and any lift $\rho$ of $\bar{\rho}$ as in condition (2), the socle of $\pi^{\circ} \otimes_{\mathcal{O}} k^{\prime}$ is the unique absolutely irreducible generic representation $\bar{\pi}^{\text {gen }}$ of $\mathrm{GL}_{n}(F)$ whose supercuspidal support corresponds to $\bar{\rho}^{\mathrm{ss}}$ under the mod $p$ semisimple local Langlands correspondence of Vigneras [5]. Thus $\pi^{\circ} \otimes_{\mathcal{O}} k^{\prime}$ embeds in the essentially AIG envelope env $\left(\bar{\pi}^{\text {gen }}\right)$, so that env $\left(\bar{\pi}^{\text {gen }}\right)$ satisfies conditions (1) and (2) of the theorem, but may be too large. One obtains $\bar{\pi}(\bar{\rho})$ by taking the sum, inside env $\left(\bar{\pi}^{\text {gen }}\right) \otimes_{k} \bar{k}$, of the subobjects $\pi^{\circ} \otimes_{\mathcal{O}} \bar{k}$ over all lifts $\rho$ as in (2), and descending from $\bar{k}$ to $k$.

When $n=2$ and $p$ is odd, this perspective is all that one needs to explicitly describe the modified mod $p$ local Langlands correspondence. In particular, when $\bar{\rho}^{\mathrm{ss}}$ is not a twist of the direct sum $1 \oplus \bar{\omega}$, where $\bar{\omega}$ is the $\bmod p$ cyclotomic character, then $\operatorname{env}\left(\bar{\pi}^{\text {gen }}\right)$ is irreducible, and thus the inclusions $\bar{\pi}^{\text {gen }} \subseteq \bar{\pi}(\bar{\rho}) \subseteq \operatorname{env}\left(\bar{\pi}^{\text {gen }}\right)$ are all equalities. When $\bar{\rho}^{\text {ss }}$ is a twist of $1 \oplus \bar{\omega}$, the situation is slightly more complicated, but still easy as long as $q$ is not congruent to \pm 1 modulo $p$. We will cover this so-called "banal" case in Section 2, below.

In Section 3, we cover the case when $q$ is congruent to -1 modulo $p$, and $\bar{\rho}^{\mathrm{ss}}$ is a twist of $1 \oplus \omega$. This case was worked out independently by Emerton in unpublished work. It is similar to the case when $q$ is not congruent to \pm 1 modulo $p$, but is slightly more complicated because env $\left(\bar{\pi}^{\text {gen }}\right)$ has length 3 instead of 2 .

The remaining sections are devoted to the case when $q$ is congruent to 1 modulo $p$, and $\bar{\rho}^{\text {ss }}$ is a twist of $1 \oplus \bar{\omega}$. (Note that $\bar{\omega}$ is trivial in this setting.) This case is the most difficult because in this setting there is a one-parameter family of representations $\bar{\rho}$ whose semisimplification is trivial. It turns out (Theorem 5.8) in this case that the modified mod $p$ local Langlands correspondence is sensitive enough to 
distinguish between these distinct extensions. This is in stark contrast to the situation in characteristic zero, where the Breuil-Schneider correspondence is insensitive to Frobenius-semisimplification. This "extra sensitivity" is quite striking and would be worth investigating in situations where $n$ is greater than two.

We omit from this discussion the case when $p=2$, which is significantly more complicated than the cases discussed here. (In particular, in that setting, $\operatorname{env}\left(\bar{\pi}^{\text {gen }}\right)$ has length five, and classifying the possible lifts of the trivial representation $\bar{\rho}$ becomes rather more complicated.)

\section{The banal case}

In this section and the next, we write $G$ for $\mathrm{GL}_{2}(F)$, for conciseness. Let $B$ be the standard (upper triangular) Borel subgroup of $G$, and $T$ the standard maximal torus.

This section will be concerned with the case in which $q$ is not congruent to 1 or -1 modulo $p$, and $\bar{\rho}^{\text {ss }}$ is a twist of $1 \oplus \bar{\omega}$. Since the modified mod $p$ local Langlands correspondence is compatible with twisting, we may assume that $\bar{\rho}^{\mathrm{ss}}$ is equal to $1 \oplus \bar{\omega}$. The semisimple mod $p$ local Langlands correspondence of Vigneras then shows that $\bar{\pi}^{\text {gen }}$, and indeed every Jordan-Hölder constituent of env $\left(\bar{\pi}^{\text {gen }}\right)$, has supercuspidal support given by the two characters ||$^{ \pm \frac{1}{2}}$. Thus every Jordan-Hölder constituent of $\operatorname{env}\left(\bar{\pi}^{\text {gen }}\right)$ is also a Jordan-Holder constituent of the normalized parabolic induction $i_{B}^{G}||^{\frac{1}{2}} \otimes||^{-\frac{1}{2}}$. This induction is reducible and admits as a quotient the trivial representation $1_{G}$.

Let $V$ be the parabolic induction $i_{B}^{G}||^{\frac{1}{2}} \otimes||^{-\frac{1}{2}}$. In the "banal" case we are currently considering, this parabolic induction has length two. Indeed, the JordanHölder constituents of the parabolic restriction $r_{G}^{B} V$ are the two characters ||$^{\frac{1}{2}} \otimes$ $|\quad|^{-\frac{1}{2}}$ and $|\quad|^{-\frac{1}{2}} \otimes|\quad|^{\frac{1}{2}}$ of $T$; when $q$ is not 1 modulo $p$ these are non-trivial and any extension of one by the other is split.

Thus there are exactly two non-cuspidal constituents of $V$. Any additional constituent would be cuspidal but not supercuspidal, and (as is well-known) such representations do not exist in the banal setting.

We thus have an exact sequence:

$$
0 \rightarrow \mathrm{St} \rightarrow V \rightarrow 1_{G} \rightarrow 0,
$$

where the kernel St of the map $V \rightarrow 1_{G}$ is irreducible and generic. In particular we have $\bar{\pi}^{\text {gen }}=$ St.

It is not hard now to show:

Lemma 2.1. The representation $V$ is an essentially $A I G$ envelope of $\bar{\pi}^{\text {gen }}$.

Proof. Let $V^{\prime}$ be essentially AIG, with socle $\bar{\pi}^{\text {gen }}$. Then $V^{\prime}$ contains a submodule isomorphic to St, all of its other subquotients are isomorphic to $1_{G}$. Thus, $r_{G}^{B} V^{\prime}$ contains a submodule isomorphic to ||$^{\frac{1}{2}} \otimes|\quad|^{-\frac{1}{2}}$, whereas all other irreducible subquotients of $r_{G}^{B} V^{\prime}$ are isomorphic to ||$^{-\frac{1}{2}} \otimes|\quad|^{\frac{1}{2}}$. As there is no non-split extension of these two characters, we obtain a non-zero map: $r_{G}^{B} V^{\prime} \rightarrow||^{\frac{1}{2}} \otimes||^{-\frac{1}{2}}$. By Frobenius reciprocity, this gives us a non-zero map $V^{\prime} \rightarrow V$ which must be injective because it is a map of essentially AIG representations.

Thus for any $\bar{\rho}$ whose semisimplification is $1 \oplus \bar{\omega}$, we either have $\bar{\pi}(\bar{\rho})=$ St or $\bar{\pi}(\bar{\rho})=V$. It is easy to distinguish the two cases: 
Lemma 2.2. We have $\bar{\pi}(\bar{\rho})=V$ if, and only if, $\bar{\rho}$ is split.

Proof. If $\bar{\rho}$ is non-split, then any lift $\rho$ of $\bar{\rho}$ is a non-split extension. Then $\pi\left(\rho \otimes_{\mathcal{O}} K\right)$ is a twist of a Steinberg representation, and so its reduction modulo $p$ is isomorphic to St $\otimes_{k} k^{\prime}$. Thus, in this case $\bar{\pi}(\bar{\rho})=$ St.

On the other hand, if $\bar{\rho}$ is split, then $\bar{\rho}$ admits a lift $\rho$ that is also split. The corresponding $\pi\left(\rho \otimes_{\mathcal{O}} K\right)$ is a parabolic induction, and thus (since parabolic induction commutes with reduction $\bmod p), \pi\left(\rho \otimes_{\mathcal{O}} K\right)$ contains a lattice whose reduction mod $p$ is isomorphic to $V \otimes_{k} k^{\prime}$. Thus in this case, $\bar{\pi}(\bar{\rho})=V$.

\section{3. $q \equiv-1 \bmod p$}

Now suppose that $q$ is congruent to -1 modulo $p$, and that $\bar{\rho}^{\mathrm{ss}}$ is isomorphic to $1 \oplus \bar{\omega}$. As in the previous section, every Jordan-Hölder constituent of $\operatorname{env}\left(\bar{\pi}^{\text {gen }}\right)$ is also a Jordan-Holder constituent of the normalized parabolic induction $i_{B}^{G}||^{\frac{1}{2}} \otimes$ ||$^{-\frac{1}{2}}$. There are three such constituents in this setting (cf. Example II.2.5 of [4]): the trivial character of $G$, the character $\mid$ | o det (which has values in \pm 1 because of our assumption on $q$ ), and a cuspidal subquotient, which is the unique generic subquotient and is thus isomorphic to $\bar{\pi}^{\text {gen }}$. More precisely, we have exact sequences:

$$
\begin{gathered}
0 \rightarrow W \rightarrow i_{B}^{G}|\quad|^{-\frac{1}{2}} \otimes|\quad|^{\frac{1}{2}} \rightarrow|\quad| \circ \text { det } \rightarrow 0, \\
0 \rightarrow 1_{G} \rightarrow W \rightarrow \bar{\pi}^{\text {gen }} \rightarrow 0,
\end{gathered}
$$

for a suitable representation $W$. Both of these sequences are non-split, as $\bar{\pi}^{\text {gen }}$ is cuspidal and thus is neither a subobject nor a quotient of any proper parabolic induction. Throughout this section we will make use of the assumption that $p$ is odd; in this case the characters $1_{G}$ and $\mid$ | det are distinct.

Lemma 3.1. Any non-split extension of $\bar{\pi}^{\text {gen }}$ by the trivial character of $G$ is isomorphic to $W$.

Proof. Let $W^{\prime}$ be such an extension. The parabolic restriction $r_{\mathrm{GL}_{2}(F)}^{B} W^{\prime}$ is isomorphic to ||$^{-\frac{1}{2}} \otimes|\quad|^{\frac{1}{2}}$. As parabolic induction is a right adjoint to parabolic restriction, this isomorphism gives rise to a non-zero map:

$$
W^{\prime} \rightarrow i_{B}^{G}|\quad|^{-\frac{1}{2}} \otimes|\quad|^{\frac{1}{2}}
$$

It is clear that such a map must be injective with image $W$.

Twisting by $\mid$ | o det we find that $W \otimes(|\quad|$ odet $)$ is the unique non-split extension of $\bar{\pi}^{\text {gen }}$ by $\mid$ | o det.

As $\bar{\pi}^{\text {gen }}$ is self-dual, it is clear that $W^{\vee}$ and $W^{\vee} \otimes(|\quad| \circ$ det $)$ are essentially AIG representations with socle $\bar{\pi}^{\text {gen }}$. It follows that the representation $V$ obtained as the pushout of the diagram:

$$
\begin{array}{ccc}
W^{\vee} & \rightarrow & V \\
\uparrow & & \uparrow \\
\bar{\pi}^{\text {gen }} & \rightarrow & W^{\vee} \otimes(|\quad| \text { odet })
\end{array}
$$

is also essentially AIG. Note that $V$ is an extension of $1_{G} \oplus(|\quad| \circ$ det $)$ by $\bar{\pi}^{\text {gen }}$.

Proposition 3.2. The representation $V$ is an essentially $A I G$ envelope of $\pi^{\text {gen }}$. 
Proof. We must show that $V$ is not properly contained in an essentially AIG representation $V^{\prime}$. Suppose there were such a $V^{\prime}$. Then (as $V^{\prime}$ is the sum of its finite length submodules, and so $V^{\prime} / V$ is as well), the socle of $V^{\prime} / V$ is non-zero. This socle contains no generic summand, and is thus isomorphic to a direct sum of characters, each of which is either trivial or isomorphic to $\mid$ o det. Observing that $V$ is isomorphic to $V \otimes\left(|\quad| \circ\right.$ det) we can ensure, twisting $V^{\prime}$ if necessary, that $V^{\prime} / V$ contains a one-dimensional subspace on which $G$ acts trivially. Let $V^{\prime \prime}$ be the preimage of this subspace under the surjection

$$
V^{\prime} \rightarrow V^{\prime} / V
$$

Then $V^{\prime \prime}$ is an essentially AIG representation containing $V$, such that $V^{\prime \prime} / V$ is the character $1_{G}$, and it suffices to show that such a representation cannot exist.

Note that $V^{\prime \prime}$ is essentially AIG, and hence its only endomorphisms are scalars. In particular the centre of $G$ acts on $V^{\prime \prime}$ by a character, and this character must be trivial since the centre of $G$ acts trivially on $\bar{\pi}^{\text {gen }}$. On the other hand, $V^{\prime \prime} / \bar{\pi}^{\text {gen }}$ is en extension of $1_{G}$ by $1_{G} \oplus(|\quad|$ odet $)$, and it is easy to see that such an extension must split if the centre of $G$ acts trivially.

Thus $V^{\prime \prime}$ is an extension of $1_{G} \oplus 1_{G} \oplus(|\quad|$ odet $)$ by $\bar{\pi}^{\text {gen }}$. Let $V^{\prime \prime \prime}$ be the preimage of $1_{G} \oplus 1_{G}$ under the quotient map

$$
V^{\prime \prime} \rightarrow 1_{G} \oplus 1_{G} \oplus(|\quad| \circ \operatorname{det}) .
$$

Then $V^{\prime \prime \prime}$ is essentially $A I G$, and we have an exact sequence:

$$
0 \rightarrow \bar{\pi}^{\text {gen }} \rightarrow V^{\prime \prime \prime} \rightarrow 1_{G} \oplus 1_{G} \rightarrow 0 .
$$

Applying the functor $\operatorname{Hom}_{G}\left(1_{G},-\right)$, we obtain a long exact sequence:

$$
\operatorname{Hom}_{G}\left(1_{G}, V^{\prime \prime \prime}\right) \rightarrow \operatorname{Hom}_{G}\left(1_{G}, 1_{G} \oplus 1_{G}\right) \rightarrow \operatorname{Ext}_{G}^{1}\left(1_{G}, \bar{\pi}^{\text {gen }}\right) .
$$

As $V^{\prime \prime \prime}$ is essentially AIG the first term of this sequence is zero, so we find that $\operatorname{Ext}_{G}^{1}\left(1_{G}, \bar{\pi}^{\text {gen }}\right)$ has dimension at least two. Applying duality to Lemma 3.1 shows that $\operatorname{Ext}_{G}^{1}\left(1_{G}, \bar{\pi}^{\text {gen }}\right)$ is only one-dimensional however, and we have arrived at a contradiction.

We now turn to understanding the modified $\bmod p$ local Langlands correspondence. If $\bar{\rho}^{\text {ss }}$ is isomorphic to $1 \oplus \bar{\omega}$, then $\bar{\rho}$ is either a non-split extension of $\bar{\omega}$ by 1 , a non-split extension of 1 by $\bar{\omega}$, or the direct sum $1 \oplus \bar{\omega}$.

By contrast, let us consider the representations $\rho: G_{F} \rightarrow \mathrm{GL}_{2}(\mathcal{O})$ whose $\bmod$ $p$ reduction has semisimplification $1 \oplus \bar{\omega}$. For each such representation, let $\tilde{\rho}$ be the representation $\rho \otimes_{\mathcal{O}} K$. There are several cases:

(1) $\tilde{\rho}$ is irreducible, in which case the reduction modulo $p$ of $\rho$ can be any of the three possibilities described above.

(2) $\tilde{\rho}$ is a non-split extension of $\chi_{1}$ by $\chi_{2}$, where the $\bmod p$ reduction of $\chi_{1}$ is trivial and the $\bmod p$ reduction of $\chi_{2}$ is $\bar{\omega}$. In this case, the $\bmod p$ reduction of $\rho$ has a subrepresentation isomorphic to $\bar{\omega}$, and thus cannot be a non-split extension of $\bar{\omega}$ by 1 .

(3) $\tilde{\rho}$ is a non-split extension of $\chi_{2}$ by $\chi_{1}$, where the $\bmod p$ reduction of $\chi_{1}$ is trivial and the $\bmod p$ reduction of $\chi_{2}$ is $\bar{\omega}$. In this case, the mod $p$ reduction of $\rho$ cannot be a non-split extension of 1 by $\bar{\omega}$. 
(4) $\tilde{\rho}$ is a direct sum of two characters. In this case, the $\bmod p$ reduction of $\rho$ must be the direct sum $1 \oplus \bar{\omega}$.

It is straightforward to describe $\pi(\tilde{\rho})$, and the reduction $\pi(\tilde{\rho})^{\circ} \otimes_{\mathcal{O}} k^{\prime}$, in each of the above cases. Indeed, it follows easily from the definition of $\pi(\tilde{\rho})$ in Section 4.2 of [3] that for a two-dimensional representation $\tilde{\rho}$ of $G_{F}$ over $K, \pi(\tilde{\rho})$ is the irreducible representation of $G$ attached to $\tilde{\rho}$ via local Langlands unless the latter is a character. In this case $\pi(\tilde{\rho})$ is a reducible parabolic induction, whose unique quotient is the character attached to $\tilde{\rho}$ via local Langlands. This determines $\pi(\tilde{\rho})$ uniquely.

We have four cases to consider:

(1) $\pi(\tilde{\rho})$ is irreducible and cuspidal. Moreover (as $\tilde{\rho}$ is integral), the central character of $\pi(\tilde{\rho})$ is integral. It follows, for instance by the results of Sections II.4.12-13 of [4], that $\pi(\tilde{\rho})$ is integral. Since parabolic restriction commutes with reduction modulo $p$, the representation $\pi(\tilde{\rho})^{\circ} \otimes_{\mathcal{O}} k^{\prime}$ is cuspidal. The only cuspidal subrepresentation of $\operatorname{env}\left(\bar{\pi}^{\text {gen }}\right)$ is $\bar{\pi}^{\text {gen }}$ itself, so we must have an isomorphism of $\pi(\tilde{\rho})^{\circ} \otimes_{\mathcal{O}} k^{\prime}$ with $\bar{\pi}^{\text {gen }} \otimes_{k} k^{\prime}$.

(2) $\pi(\tilde{\rho})$ is a twist of the Steinberg representation by a character that is trivial modulo $p$. The reduction mod $p$ of $\pi(\tilde{\rho})$ then has two Jordan-Hölder constituents, isomorphic to $\bar{\pi}^{\text {gen }}$ and $|\quad| \circ$ det. In particular, $\pi(\tilde{\rho})^{\circ} \otimes_{\mathcal{O}} k^{\prime}$ is a non-split extension of $(|\quad| \circ$ det $) \otimes_{k} k^{\prime}$ by $\bar{\pi}^{\text {gen }} \otimes_{k} k^{\prime}$, and is thus isomorphic to $W^{\vee} \otimes(|\quad|$ o det $) \otimes k^{\prime}$.

(3) $\pi(\tilde{\rho})$ is a twist of the Steinberg representation by a character that is congruent to | $\mid$ o det modulo $p$. In this case $\pi(\tilde{\rho})^{\circ} \otimes_{\mathcal{O}} k^{\prime}$ is isomorphic to $W^{\vee} \otimes k^{\prime}$.

(4) $\pi(\tilde{\rho})$ is a parabolic induction, that contains a lattice whose reduction modulo $p$ is $i_{B}^{G}||^{\frac{1}{2}} \otimes||^{-\frac{1}{2}}$. The reduction of $\pi(\tilde{\rho})^{\circ}$ thus has length 3 and embeds in $V \otimes_{k} k^{\prime}$, and is thus isomorphic to $V \otimes_{k} k^{\prime}$.

It is now easy to establish the modified mod $p$ local Langlands correspondence for these $\bar{\rho}$ :

Theorem 3.3. Let $\bar{\rho}$ be a representation of $G_{F}$ such that $\bar{\rho}^{\mathrm{ss}} \cong 1 \oplus \bar{\omega}$.

(1) If $\bar{\rho}=1 \oplus \bar{\omega}$, then $\bar{\pi}(\bar{\rho})$ is isomorphic to $V$.

(2) If $\bar{\rho}$ is a non-split extension of 1 by $\bar{\omega}$, then $\bar{\pi}(\bar{\rho})$ is isomorphic to $W^{\vee} \otimes$ (| | o det).

(3) If $\bar{\rho}$ is a non-split extension of $\bar{\omega}$ by 1 , then $\bar{\pi}(\bar{\rho})$ is isomorphic to $W^{\vee}$.

Proof. In case (1), $\bar{\rho}$ has a lift $\rho$ of type (4), and then $\pi(\tilde{\rho})^{\circ} \otimes_{\mathcal{O}} k^{\prime}$ is isomorphic to $V \otimes_{k} k^{\prime}$. On the other hand $\bar{\pi}(\bar{\rho})$ is contained in $V$ and $\bar{\pi}(\bar{\rho}) \otimes_{k} k^{\prime}$ contains $\pi(\tilde{\rho})^{\circ} \otimes_{\mathcal{O}} k^{\prime}$. We must thus have $\bar{\pi}(\bar{\rho})=V$.

In case (2), $\bar{\rho}$ has lifts of type (1) and (2), but not (3) or (4). Thus $\pi(\tilde{\rho})^{\circ} \otimes_{\mathcal{O}} k^{\prime}$ is contained in $W^{\vee} \otimes(|\quad| \circ$ det $) \otimes_{\mathcal{O}} k^{\prime}$, and the two are sometimes equal. We must thus have $\bar{\pi}(\bar{\rho})=W^{\vee} \otimes(|\quad| \circ$ det$)$. Case (3) follow from case (2) by twisting by | $\mid$ o det.

\section{Lattices in direct sums of characters}

Before we turn to the case where $q \equiv 1 \bmod p$, we need a technical result. For this section, let $G$ be an arbitrary locally profinite group. Let $\mathcal{O}$ be a discrete valuation ring with residue field $k$, uniformizer $\varpi$, and field of fractions $K$. 
Let $\chi_{1}$ and $\chi_{2}$ be two distinct characters $G \rightarrow \mathcal{O}^{\times}$that are trivial modulo $\varpi$. We will attach a class $\sigma\left(\chi_{1}, \chi_{2}\right)$ in $H^{1}(G, k)$ (where $G$ acts trivially on $k$ ) to this pair of characters. Let $a$ be the largest integer such that $\chi_{1}$ and $\chi_{2}$ are congruent modulo $a$. Then for $g$ in $G$ we define $\sigma\left(\chi_{1}, \chi_{2}\right)_{g}$ to be the reduction modulo $\varpi$ of the element $\frac{1}{\varpi^{a}}\left(\chi_{1}(g)-\chi_{2}(g)\right)$.

Note that $H^{1}(G, k)$ is isomorphic to $\operatorname{Ext}_{G}^{1}\left(1_{G}, 1_{G}\right)$, where $1_{G}$ denotes the trivial character of $G$ with values in $k$. There is thus a bijection between lines in $H^{1}(G, k)$ and non-split extensions of $1_{G}$ by $1_{G}$. This bijection can be made entirely explicit as follows: let $E$ be such an extension, let $e_{1}$ span the invariant line in $E$, and complete this to a $k$-basis $\left\{e_{1}, e_{2}\right\}$ of $E$. For any $g, g e_{2}-e_{2}$ is equal to $\sigma_{g} e_{1}$ for some $\sigma_{g}$ in $k$; the cocycle $g \mapsto \sigma_{g}$ represents a class in $H^{1}(G, k)$ that is non-trival because $E$ is not split. A different choice of basis $\left\{e_{1}, e_{2}\right\}$ changes $\sigma$ by a non-zero scalar, and this gives the desired bijection of extensions $E$ with lines in $H^{1}(G, k)$.

Our goal in this section is to interpret the class $\sigma\left(\chi_{1}, \chi_{2}\right)$ in terms of this isomorphism. Let $L$ be a free $\mathcal{O}$-module of rank two, with basis $e_{1}$ and $e_{2}$. Define an action of $G$ on $L$ by $g e_{1}=\chi_{1}(g) e_{1}$ and $g e_{2}=\chi_{2}(g) e_{2}$. Let $L^{\prime}$ be a $G$-stable $\mathcal{O}$-lattice in $L \otimes K$. Then $L^{\prime} / \varpi L^{\prime}$ is an extension of $1_{G}$ by $1_{G}$, and we have:

Proposition 4.1. Suppose $L^{\prime} / \varpi L^{\prime}$ is non-split. Then $\sigma\left(\chi_{1}, \chi_{2}\right)$ generates the line in $H^{1}(G, k)$ corresponding to the extension $L^{\prime} / \varpi L^{\prime}$.

Proof. Since replacing $L^{\prime}$ with $\varpi L^{\prime}$ does not change the extension $L^{\prime} / \varpi L^{\prime}$, we may assume without loss of generality that $L \subset L^{\prime}$ but $L \nsubseteq \varpi L^{\prime}$. Then the map

$$
L / \varpi L \rightarrow L^{\prime} / \varpi L^{\prime}
$$

has one-dimensional image. Swapping $e_{1}$ and $e_{2}$ (and thus $\chi_{1}$ and $\chi_{2}$ ) if necessary we may assume that $e_{1}$ generates the image of $L / \varpi L$ in $L^{\prime} / \varpi L^{\prime}$. (Note that this only changes $\sigma\left(\chi_{1}, \chi_{2}\right)$ by a sign.)

Since $e_{1}$ is non-zero in $L^{\prime} / \varpi L^{\prime}$ we may complete it to a basis $\left\{e_{1}, e_{3}\right\}$ of $L^{\prime}$. Let $b$ be the smallest integer greater than zero such that $\varpi^{b} e_{3}$ lies in $L$, and write $\varpi^{b} e_{3}=$ $\alpha e_{1}+\beta e_{2}$ for $\alpha, \beta \in \mathcal{O}$. We then have $g e_{3}=\chi_{2}(g) e_{3}+\frac{1}{\varpi^{b}} \alpha\left(\chi_{1}(g)-\chi_{2}(g)\right) e_{1}$. Note that by assumption the coefficient of $e_{1}$ lies in $\mathcal{O}$, as $L^{\prime}$ is $G$-stable.

Let $\bar{e}_{1}, \bar{e}_{3}$ be the images of $e_{1}$ and $e_{3}$ in $L^{\prime} / \varpi L^{\prime}$. The action of $G$ fixes $\bar{e}_{1}$, whereas $g \bar{e}_{3}=\bar{e}_{3}+\sigma_{g} \bar{e}_{1}$, where $\sigma_{g}$ is the reduction modulo $\varpi$ of $\frac{1}{\varpi b} \alpha\left(\chi_{1}(g)-\chi_{2}(g)\right)$. As $L^{\prime} / \varpi L^{\prime}$ is non-split, $\sigma_{g}$ is non-zero for some $g$, and thus $\frac{1}{\varpi^{b}} \alpha$ must lie in $\frac{1}{\varpi^{a}} \mathcal{O}^{\times}$. Thus, $\sigma$ is a scalar multiple of $\sigma\left(\chi_{1}, \chi_{2}\right)$ as claimed.

\section{5. $q \equiv 1 \bmod p$}

We now consider the case in which $q \equiv 1 \bmod p$. In this case, $\bar{\omega}$ is the trivial character, and, so, up to twist, the only case it remains to consider is when $\bar{\rho}^{\mathrm{ss}}$ is the twodimensional trivial representation of $G=\mathrm{GL}_{2}(F)$. As above, we begin by computing the appropriate essentially AIG envelope.

In this setting every subquotient of the essentially AIG envelope that contains $\bar{\pi}(\bar{\rho})$ has supercuspidal support given by two copies of the trivial character, and is thus isomorphic to a subquotient of the parabolic induction $i_{B}^{G} 1_{T}$, where $B \subset G$ is the standard Borel, $T$ is the standard torus, and $1_{T}$ is the trivial character of the torus over $k$. 
We continue to assume that $p$ is odd. This means that $i_{B}^{G} 1_{T}$ has length two: as always, $r_{G}^{B} i_{B}^{G} 1_{T}$ has length two, so any further Jordan-Hölder constituents must be cuspidal (but not supercuspidal). It is well-known by work of Vigneras, however, that $G$ has such representations only if $q \equiv-1 \bmod p$.

The induction $i_{B}^{G} 1_{T}$ thus has two Jordan-Hölder constituents: the trivial character $1_{G}$, and the Steinberg representation St of $G$ over $k$. We thus have $\bar{\pi}^{\text {gen }}=$ St.

Lemma 5.1. There is an isomorphism:

$$
i_{B}^{G} 1_{T} \cong 1_{G} \oplus \text { St. }
$$

Proof. The restrictions $r_{G}^{B} 1_{G}$ and $r_{G}^{B}$ St are both isomorphic to the trivial character $1_{T}$, because the norm character || is trivial. We thus have:

$$
\begin{aligned}
& \operatorname{Hom}_{G}\left(1_{G}, i_{B}^{G} 1_{T}\right)=\operatorname{Hom}_{T}\left(1_{T}, 1_{T}\right) \\
& \operatorname{Hom}_{G}\left(\mathrm{St}, i_{B}^{G} 1_{T}\right)=\operatorname{Hom}_{T}\left(1_{T}, 1_{T}\right)
\end{aligned}
$$

and the claim follows.

Lemma 5.2. The space $\operatorname{Ext}_{G}^{1}\left(1_{G}, \mathrm{St}\right)$ is two-dimensional.

Proof. First note that, by applying the two isomorphic functors $\operatorname{Hom}_{G}\left(1_{G}, i_{B}^{G}-\right)$ and $\operatorname{Hom}_{T}\left(1_{T},-\right)$ to an injective resolution of $1_{T}$, and observing that (as a right adjoint of an exact functor) $i_{B}^{G}$ takes injectives to injectives, we obtain an isomorphism:

$$
\operatorname{Ext}_{G}^{1}\left(1_{G}, i_{B}^{G} 1_{T}\right) \cong \operatorname{Ext}_{T}^{1}\left(1_{T}, 1_{T}\right) .
$$

The space $\operatorname{Ext}_{T}^{1}\left(1_{T}, 1_{T}\right)$ is four dimensional. On the other hand

$$
\operatorname{Ext}_{G}^{1}\left(1_{G}, i_{B}^{G} 1_{T}\right) \cong \operatorname{Ext}_{G}^{1}\left(1_{G}, 1_{G}\right) \oplus \operatorname{Ext}_{G}^{1}\left(1_{G}, \mathrm{St}\right) .
$$

One easily sees that $\operatorname{Ext}_{G}^{1}\left(1_{G}, 1_{G}\right)$ is two-dimensional, and the result follows.

Let $V$ be the "universal extension" of $1_{G}$ by St, in other words the unique extension of $1_{G} \oplus 1_{G}$ by St that contains every isomorphism class of extension of $1_{G}$ by St (more prosaically $V$ may be constructed as the pushout:

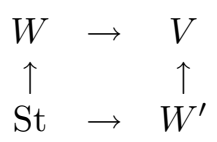

where $W$ and $W^{\prime}$ are any two nonisomorphic extensions of $1_{G}$ by St.) We then have:

Proposition 5.3. The representation $V$ is an essentially AIG envelope of St.

Proof. Suppose not. Then (just as in the $q \equiv-1 \bmod p$ case), there is an essentially AIG representation $V^{\prime}$ containing $V$ with $V^{\prime} / V$ isomorphic to $1_{G}$. The quotient $V^{\prime} / \mathrm{St}$ is an extension of $1_{G}$ by $1_{G} \oplus 1_{G}$ on which the centre of $G$ acts trivially.

We will show that such an extension is split. Indeed, such an extension is finite dimensional, and hence the action of $G$ on such an extension factors through det. The image of the centre of $G$ under det is $\left(F^{\times}\right)^{2}$. In particular, $g^{2}$ acts trivially on such an extension for any $g \in G$. On the other hand, the action of any $g$ in $G$ on this extension is unipotent, and thus has order a power of $p$. Since we are assuming that $p$ is odd, the claim follows. 
Thus $V^{\prime}$ is an extension of $1_{G} \oplus 1_{G} \oplus 1_{G}$ by St; since $\operatorname{Ext}_{G}^{1}\left(1_{G}, \mathrm{St}\right)$ is only two dimensional we must have that $1_{G}$ is a direct summand of $V^{\prime}$, contradicting the fact that $V^{\prime}$ is essentially AIG.

It will be useful to be able to classify the non-split extensions of $1_{G}$ by St. Observe:

Lemma 5.4. Let $W$ be a non-split extension of $1_{G}$ by St. Then $r_{G}^{B} W$ is a non-split extension of $1_{T}$ by $1_{T}$. (Equivalently, the map

$$
\operatorname{Ext}_{G}^{1}\left(1_{G}, \mathrm{St}\right) \rightarrow \operatorname{Ext}_{T}^{1}\left(1_{T}, 1_{T}\right)
$$

induced by $r_{G}^{B}$ is injective.)

Proof. Suppose $r_{G}^{B} W$ is split. Then $\operatorname{Hom}_{T}\left(r_{B}^{G} W, 1_{T}\right)$ is two dimensional, so, by Frobenius reciprocity, $\operatorname{Hom}_{G}\left(W, i_{B}^{G} 1_{T}\right)$ is also two-dimensional. It follows that there is a surjection of $W$ onto St, implying that $W$ must also be split.

It is not difficult to characterise the image of this map:

Lemma 5.5. Let $E$ be an extension of $1_{T}$ by $1_{T}$. Then there exists an extension $W$ of $1_{G}$ by St with $r_{G}^{B} W=E$ if, and only if, the centre $Z$ of $G$ acts trivially on $E$.

Proof. The representation $W$ is essentially AIG and so $Z$ acts on $W$ by scalars, and hence trivially. Thus the same is true of $r_{B}^{G} W$. Thus the image of the map

$$
r_{G}^{B}: \operatorname{Ext}_{G}^{1}\left(1_{G}, \mathrm{St}\right) \rightarrow \operatorname{Ext}_{T}^{1}\left(1_{T}, 1_{T}\right)
$$

is contained in the subspace $\operatorname{Ext}_{T / Z}^{1}\left(1_{T / Z}, 1_{T / Z}\right)$ of $\operatorname{Ext}_{T}^{1}\left(1_{T}, 1_{T}\right)$. This subspace is two-dimensional, as is the image of $r_{G}^{B}$, proving the claim.

Let $W_{F}$ be the Weil group of $F$. The sequence of isomorphisms: $W_{F}^{\mathrm{ab}} \cong F^{\times} \cong T / Z$ (where the last isomorphism sends $x \in F^{\times}$to the class of the diagonal matrix with entries $x$ and 1) induces a chain of isomorphisms:

$$
\operatorname{Ext}_{G_{F}}^{1}\left(1_{G_{F}}, 1_{G_{F}}\right) \cong \operatorname{Ext}_{W_{F}}^{1}\left(1_{W_{F}}, 1_{W_{F}}\right) \cong \operatorname{Ext}_{F^{\times}}^{1}\left(1_{F^{\times}}, 1_{F^{\times}}\right) \cong \operatorname{Ext}_{T / Z}^{1}\left(1_{T / Z}, 1_{T / Z}\right) \text {. }
$$

Denote the composition of these morphisms by $\phi$. We observe:

Lemma 5.6. Let $K$ be a finite extension of $\mathbb{Q}_{p}$ with uniformizer $\varpi$, residue field $k^{\prime}$ and ring of integers $\mathcal{O}$. Let $\hat{\chi}_{1}$ and $\hat{\chi}_{2}$ be distinct characters of $G_{F}$ with values in $\mathcal{O}$, whose reductions mod $\varpi$ are trivial, and let $\chi_{1}$ and $\chi_{2}$ be the corresponding characters of $F^{\times}$. Then the sequence of maps:

$$
H^{1}\left(G_{F}, 1_{G_{F}}\right) \cong \operatorname{Ext}_{G_{F}}^{1}\left(1_{G_{F}}, 1_{G_{F}}\right) \stackrel{\phi}{\rightarrow} \operatorname{Ext}_{T / Z}^{1}\left(1_{T / Z}, 1_{T / Z}\right) \cong H^{1}\left(T / Z, 1_{T / Z}\right)
$$

takes $\sigma\left(\chi_{1}, \chi_{2}\right)$ to a non-zero multiple of the class $\sigma\left(\chi_{1} \otimes \chi_{2}, \chi_{2} \otimes \chi_{1}\right)$.

Proof. This is an easy computation.

We are now in a position to describe $\bar{\pi}(\bar{\rho})$ for each $\bar{\rho}$. We first enumerate the possible lifts $\rho$ of $\bar{\rho}$. Let $\rho$ be a lift of $\bar{\rho}$ to a representation over $\mathcal{O}$, and set $\tilde{\rho}=\rho \otimes_{\mathcal{O}} K$. There are four cases:

(1) $\tilde{\rho}$ is a two-dimensional representation of $G_{F}$ on which $G_{F}$ acts via a single character $\hat{\chi}$. In this case, $\bar{\rho}$ must be trivial. 
(2) $\tilde{\rho}$ is the direct sum of two distinct characters $\hat{\chi}_{1}$ and $\hat{\chi}_{2}$ whose reductions are trivial. In this case $\bar{\rho}$ is either trivial or the unique non-split extension of $1_{G_{F}}$ by $1_{G_{F}}$ of class $\sigma\left(\hat{\chi}_{1}, \hat{\chi}_{2}\right)$.

(3) $\tilde{\rho}$ is a non-split extension of a character $\hat{\chi}$ by the character $\omega \hat{\chi}$.

(4) $\tilde{\rho}$ is a twist of the unique unramified extension of the trivial representation of $G_{F}$ over $K$ by itself. In this case $\bar{\rho}$ is either trivial or the unique unramified extension of $1_{G_{F}}$ by $1_{G_{F}}$.

The next step is to describe $\pi(\tilde{\rho})^{\circ} \otimes_{\mathcal{O}} k^{\prime}$. We first observe:

Lemma 5.7. Let $K$ be a finite extension of $\mathbb{Q}_{p}$, and let $\pi$ be the irreducible parabolic induction $i_{B}^{G} 1_{T, K}$, where $1_{T, K}$ is the one-dimensional trivial representation of $T$ over $K$. Then $r_{G}^{B} \pi$ is the unique non-split extension of $1_{T, K}$ by $1_{T, K}$ on which the action of $T$ factors through the quotient: $T \rightarrow T / Z \cong F^{\times} \rightarrow \mathbb{Z} \mathbb{Z}$.

Proof. It is clear that the extension $r_{G}^{B} \pi$ is non-split, as we have isomorphisms:

$$
K \cong \operatorname{End}_{G}(\pi) \cong \operatorname{Hom}_{T}\left(r_{G}^{B} \pi, 1_{T, K}\right)
$$

It is also clear that the action of $T$ on $r_{G}^{B} \pi$ factors through $T / Z$. On the other hand the representation $\pi$ is an irreducible representation with an Iwahori fixed vector, and it is well-known that for such $\pi$, the subgroup $\mathcal{O}^{\times} \times \mathcal{O}^{\times}$of $T$ acts trivially on $r_{G}^{B} \pi$.

We can now describe $\pi(\tilde{\rho})^{\circ} \otimes_{\mathcal{O}} k^{\prime}$ in each of the above cases.

(1) In this case $\pi(\tilde{\rho})$ is a twist of $i_{B}^{G} 1_{T, K}$, and the lemma above then implies that $\pi(\tilde{\rho})^{\circ} \otimes_{\mathcal{O}} k^{\prime}$ is the unique extension $W$ of $1_{G}$ by St such that the action of $T$ on $r_{G}^{B} W$ factors through $T \rightarrow T / Z \cong F^{\times} \rightarrow \mathbb{Z} \mathbb{Z}$.

(2) In this case $\pi(\tilde{\rho})$ is the parabolic induction $i_{B}^{G} \chi_{1} \otimes \chi_{2}$, where $\chi_{1}$ and $\chi_{2}$ are the characters of $F^{\times}$arising from $\hat{\chi}_{1}$ and $\hat{\chi}_{2}$ by local class field theory. It follows that $r_{G}^{B} \pi(\tilde{\rho})$ is the direct sum of the characters $\chi_{1} \otimes \chi_{2}$ and $\chi_{2} \otimes \chi_{1}$, and $r_{G}^{B} \pi(\tilde{\rho})^{\circ} \otimes_{\mathcal{O}} k^{\prime}$ is then the non-split extension of $1_{T}$ by $1_{T}$ of class $\sigma\left(\chi_{1} \otimes\right.$ $\left.\chi_{2}, \chi_{2} \otimes \chi_{1}\right)$.

(3) In this case $\pi(\tilde{\rho})$ is a twist of the Steinberg representation, and $\pi(\tilde{\rho})^{\circ} \otimes_{\mathcal{O}} k^{\prime}$ is isomorphic to $\mathrm{St} \otimes_{k} k^{\prime}$.

(4) In this case $\pi(\tilde{\rho})$ is a twist of $i_{B}^{G} 1_{T, K}$, and the same discussion as in case (1) applies.

Theorem 5.8. If $\bar{\rho}$ is trivial, then $\bar{\pi}(\bar{\rho})=V$. On the other hand, if $\bar{\rho}$ is the nonsplit extension of $1_{G_{F}}$ by $1_{G_{F}}$ represented by $\sigma \in \operatorname{Ext}_{G_{F}}^{1}\left(1_{G_{F}}, 1_{G_{F}}\right)$, then $\bar{\pi}(\bar{\rho})$ is the unique nonsplit extension of $1_{G}$ by St such that $r_{G}^{B} \bar{\pi}(\bar{\rho})$ represents the class $\phi(\sigma)$ in $\operatorname{Ext}_{T}^{1}\left(1_{T}, 1_{T}\right)$.

Proof. If $\bar{\rho}$ is trivial, then $\bar{\rho}$ has lifts of type (2) above for an arbitrary choice of $\hat{\chi}_{1}$ and $\hat{\chi}_{2}$. Thus $\pi(\tilde{\rho})^{\circ} \otimes_{\mathcal{O}} k^{\prime}$ can be an arbitrary non-split extension of $1_{G}$ by St. As $\bar{\pi}(\bar{\rho}) \otimes_{k} k^{\prime}$ must contain all of these extensions, and is contained in $V$, we must have $\bar{\pi}(\bar{\rho})=V$.

If $\bar{\rho}$ is non-trivial and ramified, then $\bar{\rho}$ has lifts of type (2) and possibly (3), but not (1) or (4). If $\tilde{\rho}$ is a lift of type (3) then $\pi(\tilde{\rho})^{\circ} \otimes_{\mathcal{O}} k$ is isomorphic to St and thus tells us nothing about $\bar{\pi}(\bar{\rho})$. On the other hand, if $\rho=\hat{\chi}_{1} \oplus \hat{\chi}_{2}$ is a lift of type (2), we have $\sigma=\sigma\left(\hat{\chi}_{1}, \hat{\chi}_{2}\right)$. Then $\pi(\tilde{\rho})^{\circ} \otimes_{\mathcal{O}} k^{\prime}$ is the extension of $1_{G}$ by St corresponding 
to the class $\sigma\left(\chi_{1} \otimes \chi_{2}, \chi_{2} \otimes \chi_{1}\right)$ in $\operatorname{Ext}_{T}^{1}\left(1_{T}, 1_{T}\right)$. This class is a nonzero multiple of $\phi(\sigma)$. It is thus clear that $\bar{\pi}(\bar{\rho})$ is the extension corresponding to $\phi(\sigma)$ as claimed.

If $\bar{\rho}$ is non-trivial but unramified, the discussion of the previous paragraph applies but one must also consider lifts of type (4). It suffices to check that these produce the same extension of $1_{G}$ by $\mathrm{St}$ as the lifts of type $(2)$; that is, that when $\sigma$ is the class attached to an unramified non-split extension of $1_{G_{F}}$ by $1_{G_{F}}$, then $\phi(\sigma)$ corresponds to the extension of $1_{T}$ by $1_{T}$ on which the action of $T$ factors through $T \rightarrow T / Z \cong F^{\times} \rightarrow \mathbb{Z} \mathbb{Z}$. This is a straightforward calculation.

\section{Acknowledgments}

The results in this paper grew out of a series of discussions with Matthew Emerton, and I am indebted to him for his ideas and suggestions. The paper was partially supported by NSF grant DMS-1161582.

\section{References}

[1] C. Breuil and P. Schneider, First steps towards p-adic Langlands functoriality, J. Reine Agnew. Math. 610 (2007) 149-180.

[2] M. Emerton, Local-global compatibility in the p-adic Langlands program for $\mathrm{GL}_{2 / \mathbb{Q}}$. Preprint, 2011

[3] M. Emerton and D. Helm, The local Langlands correspondence for $\mathrm{GL}_{n}$ in families. Submitted, arXiv: 1104.0321

[4] M.-F. Vignéras, Représentations $l$-modulaires d'un groupe réductif $p$-adique avec $l \neq p$, Progress in Mathematics 137, Birkhuser Boston, Boston (1996).

[5] - Correspondance locale de Langlands semi-simple pour $\operatorname{GL}(n, F)$ modulo $\ell \neq p$, Invent. Math. 144 (2001) 177-223.

Department of Mathematics, University of Texas at Austin, 1 University Station, C1200 Austin, Texas 78712, USA

E-mail address: d.helm@imperial.ac.uk 